\title{
Analysis of Non- Identical System with Two Types of Failure Using Discrete Distribution
}

\author{
JasdevBhatti $^{1}$, Ashok Chitkara ${ }^{2}$, Mohit Kakkar ${ }^{3}$ \\ ${ }^{1,3}$ (Research Scholar, Chitkara University, Punjab, India) \\ ${ }^{2}$ (Chancellor, Chitkara University, Punjab, India)
}

\begin{abstract}
In this paper the two non-identical operative parallel cold standby systems has been analyzed by using regenerative point technique.The concept of inspection policy has been introduced for failed automatic unit for detecting the kind of failures (major or minor) after which it get repaired by some repair mechanism. But the manual one is free from inspection.The model has been design for the system to calculate the various important measures of reliability i.e MTSF, steady state availability, busy period of repairman and inspector, profit function using discrete distribution and regenerative point techniques. Profit function and MTSF are also analyzed graphically.
\end{abstract}

Keywords- Geometric distribution, Regenerating point technique, MTSF, Availability, Busy period and Profit function.

\section{INTRODUCTION}

Reliability deals with the development of new techniques which helps the manufacturers to increase their system effectiveness by minimizing system failures and its maintenance costs. In reliability analysis many researchers had analyzed many systems by using continuous distribution. Aggarwal (2010) had analyzed the two units in cold standby system by considering the two types of failure and single repair time to be exponentially distributed. Said, Salah, Sherbeny (2005) had analyzed two unit cold standby system with preventive maintenance and random change in units. In this paper they introduced the concept of inspection for the repaired unit to decide whether the repair is satisfactory. Haggag (2009) had analyzed two dissimilar unit cold stanby systems with three different failure and preventive maintenance. Thus many reliability models are analyzed by using continuous distribution as it was considered to be an accurate one when the observed data were found to be large. But in case the data was found to be small, continuous distribution might not adequately describe a discrete random variable. Then one has to deal with discrete distribution to obtain the various reliability measures of the system effectiveness.

In the field of reliability using discrete distribution Bhardwaj (2009) had analyzed two unit redundant systems with imperfect switching and connection time.He also analysed two identical cold unit standby systems with discrete distribution and two types of failure.Gupta (2007) had also studied two identical unit parallel systems with Geometric failure and repair time distributions. In both reliability model analysis using conitinous and discrete distribution no one has given importance for inspection of failure when there are more than one failure

Now in this paper, two non-identical coldstandby system was anzlyzed by introducing the concept of inspection policy for detecting the two types of failure where inspection and repair time are taken as geometric distribution. Initially automatic unit is in operative and manual unit is in cold standby. On the failure of an automatic unit, an inspection is being done first to detect the one out of two types (minor or major) of failures to avoid any confussion for repairman. But no such inspection policy has been discussed for the manual one. In case both the units were together in failed condition then the preference will be given to the manual one on the automatic unit. The repairman time taken by minor is less as compaired to major.

The model is analysed stochastically and the expressions for the various reliability measures of system effectiveness such as mean time to system failure, steady state availability, and busy period for both inspector and repairman were obtained.Graphs were also been drawn to analysed the behavior of MTSF and profit function with respect to repair and failure rate.

\section{MODEL DESCRIPTION}

The following assumptions are associated with the model:

- A system consists of two non-identical cold standby units arranged in a parallel network.

- Initially automatic unit is in operative condition and the other manual one is in cold standby. 
- Upon the failure of an automatic unit, the manual unit becomes operative instantaneously.

- The system is assumed to be in the failed state when both (automatic and manual) units together were in failed conditions whether the cause of failure is major or minor.

- Inspection policy is being introduced for inspecting the failed automatic unit to inspect the kind of failure (minor or major).

- A single repairman is available to repair both types of failed unit whether the cause is major or minor one. Preference will be given to the manual failed unit on the automatic one.

- A repaired unit's works as good as new.

\section{II.1 Nomenclature}

\begin{tabular}{|l|l|l|}
\hline $\mathrm{O}$ & $:$ & Unit is in operative mode \\
\hline $\mathrm{S}$ & $:$ & Unit is in standby mode \\
\hline $\mathrm{A}_{\mathrm{o}} / \mathrm{M}_{\mathrm{o}}$ & $:$ & Automatic / Manual unit is in operative mode. \\
\hline $\mathrm{M}_{\mathrm{s}}$ & $:$ & Manual unit is in standby mode. \\
\hline $\mathrm{A}_{\mathrm{i}}$ & $:$ & Automatic unit is in failure mode and under inspection. \\
\hline $\mathrm{A}_{\mathrm{mr}} / \mathrm{A}_{\mathrm{Mr}}$ & $:$ & Automatic unit is in failure mode (minor \& major) and under repair. \\
\hline $\mathrm{A}_{\mathrm{mw}} / \mathrm{A}_{\mathrm{Mw}}$ & $:$ & $\begin{array}{l}\text { Automatic unit is in failure mode (minor \& major) and waiting for } \\
\text { repair. }\end{array}$ \\
\hline $\mathrm{M}_{\mathrm{r}}$ & & Manual unit is in failure mode (minor) and under repair. \\
\hline $\mathrm{a}$ & $:$ & Probability that unit goes to failed state with minor failure. \\
\hline $\mathrm{b}$ & $:$ & Probability that unit goes to failed state with major failure. \\
\hline $\mathrm{p}_{1 /} \mathrm{q}_{1}$ & $:$ & Probability that automatic unit goes to failed state. \\
\hline $\mathrm{p}_{2} / \mathrm{q}_{2}$ & $:$ & Probability of the failed unit to be inspected satisfactory or not. \\
\hline $\mathrm{p}_{3} / \mathrm{q}_{3}$ & $:$ & Probability that manual unit goes to failed state. \\
\hline $\mathrm{r}$ & $:$ & Failed unit ( automatic or manual ) is under repair. \\
\hline $\mathrm{q}_{\mathrm{ij}}(\mathrm{t}) / \mathrm{Q}_{\mathrm{ij}}(\mathrm{t})$ & $:$ & $\begin{array}{l}\text { p.d.f and c.d.f of first passage time from regenerative state } \mathrm{i} \text { to } \\
\text { regenerative state } \mathrm{j} .\end{array}$ \\
\hline $\mathrm{P}_{\mathrm{ij}}(\mathrm{t})$ & $:$ & Steady state transition probability from state $\mathrm{S}_{\mathrm{i}}$ to $\mathrm{S}_{\mathrm{j}}$. \\
\hline$\mu_{i}$ & $:$ & Mean sojourn time in state $\mathrm{S}_{\mathrm{i}}$. \\
\hline
\end{tabular}

"Table 1: Nomenclature"

\section{Up States}

$\mathrm{S}_{0} \equiv\left(\mathrm{A}_{\mathrm{O}}, \mathrm{M}_{\mathrm{S}}\right), \quad \mathrm{S}_{1} \equiv\left(\mathrm{A}_{\mathrm{i}}, \mathrm{M}_{\mathrm{O}}\right), \quad \mathrm{S}_{2} \equiv\left(\mathrm{A}_{\mathrm{mr}}, \mathrm{M}_{\mathrm{O}}\right)$,

$\mathrm{S}_{3} \equiv\left(\mathrm{A}_{\mathrm{Mr}}, \mathrm{M}_{\mathrm{O}}\right), \quad \mathrm{S}_{7} \equiv\left(\mathrm{A}_{\mathrm{O}}, \mathrm{M}_{\mathrm{r}}\right)$

\section{Down State}

$S_{4} \equiv\left(A_{m w}, M_{r}\right), \quad S_{5} \equiv\left(A_{M W}, M_{r}\right), S_{6} \equiv\left(A_{i}, M_{r}\right)$. 


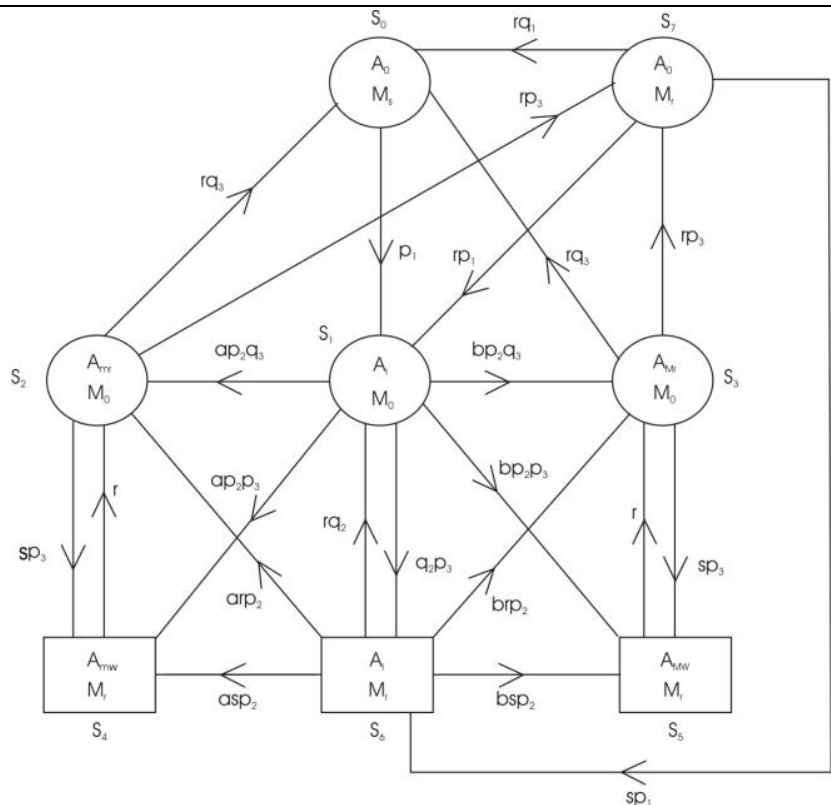

"Figure 1: Transition Diagram"

III. TRANSITION PROBABILITIES AND SOJOURN TIMES

$$
\begin{aligned}
\mathrm{Q}_{01}(\mathrm{t})=\frac{p_{1}\left[1-q_{1}{ }^{(t+1)}\right]}{1-q_{1}} & \mathrm{Q}_{12}(\mathrm{t})=\frac{a p_{2} q_{3}\left[1-q_{2} q_{3}{ }^{(t+1)}\right]}{1-q_{2} q_{3}} \\
\mathrm{Q}_{13}(\mathrm{t})=\frac{b p_{2} q_{3}\left[1-q_{2} q_{3}{ }^{(t+1)}\right]}{1-q_{2} q_{3}} & \mathrm{Q}_{14}(\mathrm{t})=\frac{a p_{2} p_{3}\left[1-q_{2} q_{3}{ }^{(t+1)}\right]}{1-q_{2} q_{3}} \\
\mathrm{Q}_{15}(\mathrm{t})=\frac{b p_{2} p_{3}\left[1-q_{2} q_{3}{ }^{(t+1)}\right]}{1-q_{2} q_{3}} & \mathrm{Q}_{16}(\mathrm{t})=\frac{q_{2} p_{3}\left[1-q_{2} q_{3}{ }^{(t+1)}\right]}{1-q_{2} q_{3}} \\
\mathrm{Q}_{20}(\mathrm{t})=\mathrm{Q}_{30}(\mathrm{t})=\frac{r q_{3}\left[1-s q_{3}{ }^{(t+1)}\right]}{1-s q_{3}} & \mathrm{Q}_{24}(\mathrm{t})=\mathrm{Q}_{35}(\mathrm{t})=\frac{s p_{3}\left[1-s q_{3}{ }^{(t+1)}\right]}{1-s q_{3}} \\
\mathrm{Q}_{27}(\mathrm{t})=\mathrm{Q}_{37}(\mathrm{t})=\frac{r p_{3}\left[1-s q_{3}{ }^{(t+1)}\right]}{1-s q_{3}} & \mathrm{Q}_{42}(\mathrm{t})=\mathrm{Q}_{53}(\mathrm{t})=\frac{r\left[1-s^{(t+1)}\right]}{1-s} \\
\mathrm{Q}_{61}(\mathrm{t})=\frac{r q_{2}\left[1-s q_{2}{ }^{(t+1)}\right]}{1-s q_{2}} & \mathrm{Q}_{62}(\mathrm{t})=\frac{a r p_{2}\left[1-s q_{2}{ }^{(t+1)}\right]}{1-s q_{2}} \\
\mathrm{Q}_{63}(\mathrm{t})=\frac{b r p_{2}\left[1-s q_{2}{ }^{(t+1)}\right]}{1-s q_{2}} & \mathrm{Q}_{64}(\mathrm{t})=\frac{a s p_{2}\left[1-s q_{2}{ }^{(t+1)}\right]}{1-s q_{2}} \\
\mathrm{Q}_{65}(\mathrm{t})=\frac{a s p_{2}\left[1-s q_{2}{ }^{(t+1)}\right]}{1-s q_{2}} & \mathrm{Q}_{71}(\mathrm{t})=\frac{r q_{1}\left[1-s q_{1}{ }^{(t+1)}\right]}{1-s q_{1}} \\
\mathrm{Q}_{70}(\mathrm{t})=\frac{r p_{1}\left[1-s q_{1}{ }^{(t+1)}\right]}{1-s q_{1}} & \mathrm{Q}_{76}(\mathrm{t})=\frac{s p_{1}\left[1-s q_{1}{ }^{(t+1)}\right]}{1-s q_{1}}
\end{aligned}
$$

The steady state transition probabilities from state $S_{i}$ to $S_{j}$ can be obtained from

$$
P_{i j}=\lim _{t \rightarrow \infty} Q_{i j}
$$

It can be verified that

$$
\begin{aligned}
& \mathrm{P}_{01}=1, \\
& \mathrm{P}_{12}+\mathrm{P}_{13}+\mathrm{P}_{14}+\mathrm{P}_{15}+\mathrm{P}_{16}=1, \\
& \mathrm{P}_{20}+\mathrm{P}_{24}+\mathrm{P}_{27}=\mathrm{P}_{30}+\mathrm{P}_{35}+\mathrm{P}_{37}=1, \\
& \mathrm{P}_{42}=\mathrm{P}_{53}=1, \\
& \mathrm{P}_{61}+\mathrm{P}_{62}+\mathrm{P}_{63}+\mathrm{P}_{64}+\mathrm{P}_{65}=1, \\
& \mathrm{P}_{70}+\mathrm{P}_{71}+\mathrm{P}_{76}=1
\end{aligned}
$$




\subsection{Mean Sojourn Times}

Let $T_{i}$ be the sojourn time in state $S_{i}(i=0,1,2,3,4,5,6,7)$, then mean sojourn time in state $S_{i}$ is given by

so that

$$
\mu_{i}=E\left(T_{i}\right)=\sum_{t=0}^{\infty} P\left(T_{i}>t\right)
$$

$$
\begin{array}{lll}
\mu_{0}=\frac{1}{1-q_{1}}, & \mu_{1}=\frac{1}{1-q_{2} q_{3}}, & \mu_{2}=\mu_{3}=\frac{1}{1-s q_{3}}, \\
\mu_{4}=\mu_{5}=\frac{1}{1-s}, & \mu_{6}=\frac{1}{1-s q_{2}} & \mu_{7}=\frac{1}{1-s q_{1}} .
\end{array}
$$

Mean sojourn time $\left(\mathrm{m}_{\mathrm{ij}}\right)$ of the system in state $\mathrm{S}_{\mathrm{i}}$ when the system is to transit into $\mathrm{S}_{\mathrm{j}}$ is given by

$$
\mathrm{m}_{\mathrm{ij}}=\sum_{\mathrm{t}=0}^{\infty} \mathrm{t} \mathrm{q}_{\mathrm{ij}}(\mathrm{t})
$$

$\mathrm{m}_{01}=\mathrm{q}_{1} \mu_{0,}$

$\mathrm{m}_{12}+\mathrm{m}_{13}+\mathrm{m}_{14}+\mathrm{m}_{15}+\mathrm{m}_{16}=\mathrm{q}_{2} \mathrm{q}_{3} \mu_{1}$

$\mathrm{m}_{20}+\mathrm{m}_{24}+\mathrm{m}_{27}=\mathrm{m}_{30}+\mathrm{m}_{35}+\mathrm{m}_{37}=\mathrm{sq}_{3} \mu_{2}$

$\mathrm{m}_{42}=\mathrm{m}_{53}=\mathrm{s} \mu_{4}$,

$\mathrm{m}_{61}+\mathrm{m}_{62}+\mathrm{m}_{63}+\mathrm{m}_{64}+\mathrm{m}_{65}=\mathrm{sq}_{2} \mu_{6}$

$\mathrm{m}_{70}+\mathrm{m}_{71}+\mathrm{m}_{76}=\mathrm{sq}_{1} \mu_{7}$.

\section{RELIABILITY AND MEAN TIME TO SYSTEM FAILURE}

Let $R_{i}(t)$ be the probability that system works satisfactorily for atleast $t$ epochs 'cycles' when it is initially started from operative regenerative state $\mathrm{S}_{\mathrm{i}}(\mathrm{i}=0,1,3,7)$.

$\mathrm{R}_{0}(\mathrm{t})=\mathrm{Z}_{0}(\mathrm{t})+\mathrm{q}_{01}(\mathrm{t}-1) \odot \mathrm{R}_{1}(\mathrm{t}-1)$.
$\mathrm{R}_{1}(\mathrm{t})=\mathrm{Z}_{1}(\mathrm{t})+\mathrm{q}_{12}(\mathrm{t}-1) \odot \mathrm{R}_{2}(\mathrm{t}-1)+\mathrm{q}_{13}(\mathrm{t}-1) \odot \mathrm{R}_{3}(\mathrm{t}-1)$.
$\mathrm{R}_{2}(\mathrm{t})=\mathrm{Z}_{2}(\mathrm{t})+\mathrm{q}_{20}(\mathrm{t}-1) \odot \mathrm{R}_{0}(\mathrm{t}-1)+\mathrm{q}_{27}(\mathrm{t}-1) \odot \mathrm{R}_{7}(\mathrm{t}-1)$.
$\mathrm{R}_{3}(\mathrm{t})=\mathrm{Z}_{3}(\mathrm{t})+\mathrm{q}_{30}(\mathrm{t}-1) \odot \mathrm{R}_{0}(\mathrm{t}-1)+\mathrm{q}_{37}(\mathrm{t}-1) \odot \mathrm{R}_{7}(\mathrm{t}-1)$.
$\mathrm{R}_{7}(\mathrm{t})=\mathrm{Z}_{7}(\mathrm{t})+\mathrm{q}_{70}(\mathrm{t}-1) \odot \mathrm{R}_{0}(\mathrm{t}-1)+\mathrm{q}_{71}(\mathrm{t}-1) \odot \mathrm{R}_{1}(\mathrm{t}-1)$.

Taking geometric transformation on both sides, we get

$$
R_{\mathrm{o}}(h)=\frac{N_{1}(h)}{D_{1}(h)}
$$

The mean time to system failure is

where

$$
\mu_{\mathrm{i}}=\lim _{\mathrm{h} \rightarrow 1} \frac{N_{1}(\mathrm{~h})}{\mathrm{D}_{1}(\mathrm{~h})}-1=\frac{\mathrm{N}_{1}}{\mathrm{D}_{1}}
$$

$\mathrm{N}_{1}=\mu_{0}+\mu_{1}-\left(\mathrm{P}_{12}+\mathrm{P}_{13}\right)\left(\mu_{0} \mathrm{P}_{27} \mathrm{P}_{71}-\mu_{2}-\mu_{7} \mathrm{P}_{27}\right)$.

$\mathrm{D}_{1}=1-\left(\mathrm{P}_{12}+\mathrm{P}_{13}\right)\left(1-\mathrm{P}_{24}-\mathrm{P}_{27} \mathrm{P}_{76}\right)$.

\section{AVAILABILITY ANALYSIS}

Let $A_{i}(t)$ is the probability that the system is up at epoch $t$ when it is initially started from regenerative state $S_{i}$. By simple probabilistic argument the following recurrence relations are obtained.

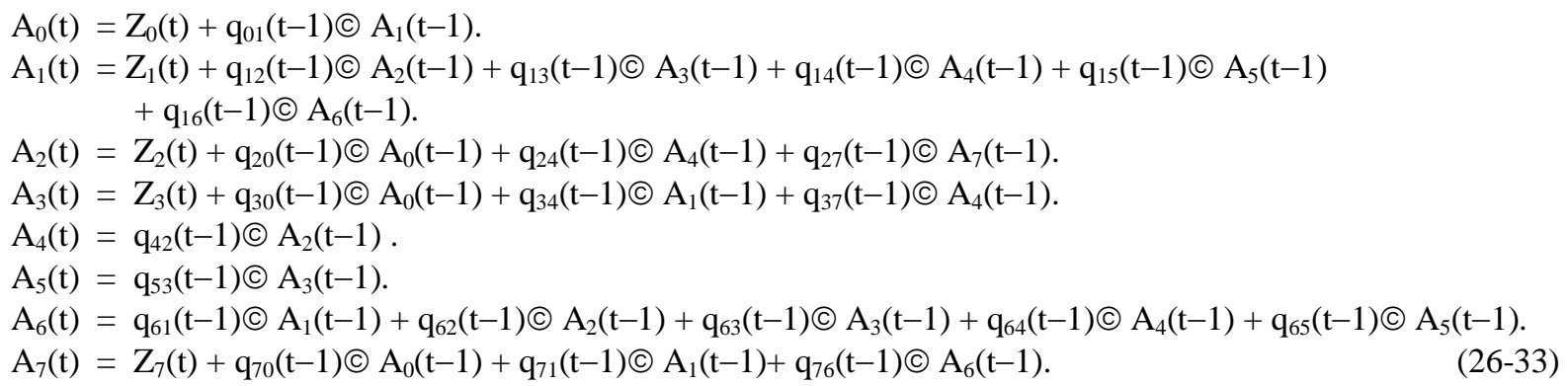


By taking geometric transformation and solving the equation

$$
A_{\mathrm{o}}(h)=\frac{N_{2}(h)}{D_{2}(h)}
$$

and

$$
Z_{i}(\mathrm{~h})=\mu_{\mathrm{i}}
$$

The steady state availability of the system is given by

$$
A_{0}=\lim _{t \rightarrow \infty} A_{0}(t)
$$

Hence, by applying ' $L$ ' Hospital Rule, we get

where

$$
A_{0}=-\frac{N_{2}(1)}{D_{2}^{\prime}(1)}
$$

$$
\begin{aligned}
\mathrm{N}_{2}(1)= & \left.\mu_{0}\left(1-\mathrm{P}_{16} \mathrm{P}_{61}\right)\left(\mathrm{P}_{20}+\mathrm{P}_{27} \mathrm{P}_{70}\right)\right]+\mu_{1}\left[1-\mathrm{P}_{24}-\mathrm{P}_{27} \mathrm{P}_{76}\left(1-\mathrm{P}_{61}\right)\right]+\mu_{2}\left(1-\mathrm{P}_{16} \mathrm{P}_{61}\right)+\mu_{7} \mathrm{P}_{27}\left(1-\mathrm{P}_{16} \mathrm{P}_{61}\right) . \\
\mathrm{D}_{2}^{\prime}(1)= & -\left\{\left[\mathrm{q}_{1} \mu_{0}\left(1-\mathrm{P}_{16} \mathrm{P}_{61}\right)\left(\mathrm{P}_{20}+\mathrm{P}_{27} \mathrm{P}_{70}\right)\right]+\mathrm{q}_{2} \mathrm{q}_{3} \mu_{1}\left[1-\mathrm{P}_{24}-\mathrm{P}_{27} \mathrm{P}_{76}\left(1-\mathrm{P}_{61}\right)\right]+\mathrm{sq}_{3} \mu_{2}\left(1-\mathrm{P}_{16} \mathrm{P}_{61}\right)\right. \\
& +\mathrm{s \mu}_{4}\left\{\mathrm{P}_{27} \mathrm{P}_{76}\left[\left(1-\mathrm{P}_{16}\right)\left(\mathrm{P}_{64}+\mathrm{P}_{65}\right)-\left(\mathrm{P}_{14}+\mathrm{P}_{15}\right)\left(1-\mathrm{P}_{61}\right)\right]+\mathrm{P}_{16}\left[\mathrm{P}_{64}+\mathrm{P}_{65}+\mathrm{P}_{24}\left(\mathrm{P}_{62}+\mathrm{P}_{63}\right)\right]\right. \\
& \left.\left.+\mathrm{P}_{14}+\mathrm{P}_{15}+\mathrm{P}_{24}\left(\mathrm{P}_{62}+\mathrm{P}_{63}\right)\right\}+\mathrm{sq}_{2} \mu_{6}\left[\mathrm{P}_{16}\left(1-\mathrm{P}_{24}\right)+\mathrm{P}_{27} \mathrm{P}_{76}\left(1-\mathrm{P}_{16}\right)\right]+\mathrm{sq}_{1} \mu_{7} \mathrm{P}_{27}\left(1-\mathrm{P}_{16} \mathrm{P}_{61}\right)\right\}
\end{aligned}
$$

\section{BUSY PERIOD ANALYSIS}

\section{VI.1 Busy period of inspector}

Let $B_{i}(t)$ be the probability of the inspector who inspect the failed unit before being repaired by repairman. Using simple probabilistic arguments, as in case of reliability and availability analysis the following recurrence relations can be easily developed.

$\mathrm{B}_{0}(\mathrm{t})=\mathrm{q}_{01}(\mathrm{t}-1) \odot \mathrm{B}_{1}(\mathrm{t}-1)$.

$\mathrm{B}_{1}(\mathrm{t})=\mathrm{q}_{12}(\mathrm{t}-1) \odot \mathrm{B}_{2}(\mathrm{t}-1)+\mathrm{q}_{13}(\mathrm{t}-1) \odot \mathrm{B}_{3}(\mathrm{t}-1)+\mathrm{q}_{14}(\mathrm{t}-1) \odot \mathrm{B}_{4}(\mathrm{t}-1)+\mathrm{q}_{15}(\mathrm{t}-1) \odot \mathrm{B}_{5}(\mathrm{t}-1)+\mathrm{q}_{16}(\mathrm{t}-1) \odot \mathrm{B}_{6}(\mathrm{t}-1)$.

$\mathrm{B}_{2}(\mathrm{t})=\mathrm{Z}_{2}(\mathrm{t})+\mathrm{q}_{20}(\mathrm{t}-1) \odot \mathrm{B}_{0}(\mathrm{t}-1)+\mathrm{q}_{24}(\mathrm{t}-1) \odot \mathrm{B}_{4}(\mathrm{t}-1)+\mathrm{q}_{27}(\mathrm{t}-1) \odot \mathrm{B}_{7}(\mathrm{t}-1)$.

$\mathrm{B}_{3}(\mathrm{t})=\mathrm{Z}_{3}(\mathrm{t})+\mathrm{q}_{30}(\mathrm{t}-1) \odot \mathrm{B}_{0}(\mathrm{t}-1)+\mathrm{q}_{35}(\mathrm{t}-1) \odot \mathrm{B}_{5}(\mathrm{t}-1)+\mathrm{q}_{37}(\mathrm{t}-1) \odot \mathrm{B}_{7}(\mathrm{t}-1)$.

$\mathrm{B}_{4}(\mathrm{t})=\mathrm{Z}_{4}(\mathrm{t})+\mathrm{q}_{42}(\mathrm{t}-1) \odot \mathrm{B}_{2}(\mathrm{t}-1)$.

$\mathrm{B}_{5}(\mathrm{t})=\mathrm{Z}_{5}(\mathrm{t})+\mathrm{q}_{53}(\mathrm{t}-1) \odot \mathrm{B}_{3}(\mathrm{t}-1)$.

$\mathrm{B}_{6}(\mathrm{t})=\mathrm{Z}_{6}(\mathrm{t})+\mathrm{q}_{61}(\mathrm{t}-1) \odot \mathrm{B}_{1}(\mathrm{t}-1)+\mathrm{q}_{62}(\mathrm{t}-1) \odot \mathrm{B}_{2}(\mathrm{t}-1)+\mathrm{q}_{63}(\mathrm{t}-1) \odot \mathrm{B}_{3}(\mathrm{t}-1)+\mathrm{q}_{64}(\mathrm{t}-1) \odot \mathrm{B}_{4}(\mathrm{t}-1)$

$$
+\mathrm{q}_{65}(\mathrm{t}-1) \odot \mathrm{B}_{5}(\mathrm{t}-1) \text {. }
$$

$\mathrm{B}_{7}(\mathrm{t})=\mathrm{Z}_{7}(\mathrm{t})+\mathrm{q}_{70}(\mathrm{t}-1) \odot \mathrm{B}_{0}(\mathrm{t}-1)+\mathrm{q}_{71}(\mathrm{t}-1) \odot \mathrm{B}_{1}(\mathrm{t}-1)+\mathrm{q}_{76}(\mathrm{t}-1) \odot \mathrm{B}_{6}(\mathrm{t}-1)$

By taking geometric transformation and solving the equation

$$
B_{0}(h)=\frac{N_{3}(h)}{D_{2}(h)}
$$

The probability that the inspection facility is busy in inspecting the failed unit is given by

$$
\mathrm{B}_{0}=\lim _{\mathrm{t} \rightarrow \infty} \mathrm{B}_{0}(\mathrm{t})
$$

Hence, by applying 'L' Hospital Rule, we get

$$
\mathrm{B}_{0}=-\frac{\mathrm{N}_{3}(1)}{\mathrm{D}_{2}^{\prime}(1)}
$$

where

$$
\begin{aligned}
\mathrm{N}_{3}(1)= & \mu_{2}\left(1-\mathrm{P}_{16} \mathrm{P}_{61}\right)+\mu_{4}\left\{\mathrm{P}_{27} \mathrm{P}_{76}\left[\left(1-\mathrm{P}_{16}\right)\left(\mathrm{P}_{64}+\mathrm{P}_{65}\right)-\left(\mathrm{P}_{14}+\mathrm{P}_{15}\right)\left(1-\mathrm{P}_{61}\right)\right]+\mathrm{P}_{16}\left[\mathrm{P}_{64}+\mathrm{P}_{65}+\mathrm{P}_{24}\left(\mathrm{P}_{62}+\mathrm{P}_{63}\right)\right]\right. \\
& \left.+\mathrm{P}_{14}+\mathrm{P}_{15}+\mathrm{P}_{24}\left(\mathrm{P}_{62}+\mathrm{P}_{63}\right)\right\}+\mu_{6}\left[\mathrm{P}_{16}\left(1-\mathrm{P}_{24}\right)+\mathrm{P}_{27} \mathrm{P}_{76}\left(1-\mathrm{P}_{16}\right)\right]+\mu_{7} \mathrm{P}_{27}\left(1-\mathrm{P}_{16} \mathrm{P}_{61}\right) .
\end{aligned}
$$

and $\mathrm{D}_{2}^{\prime}(1)$ is the same as in availability analysis.

\section{VI.2 Busy period of repairman}

Let $B_{i}^{\prime}(t)$ be the probability that the repair facility is busy in repair of failed unit when the system initially starts from regenerative state $S_{\mathrm{i}}$. Using simple probabilistic arguments, the following recurrence relations can be easily developed.

$$
\begin{aligned}
& B_{0}^{\prime}(t)=\mathrm{q}_{01}(\mathrm{t}-1) \odot B_{1}^{\prime}(t-1) \\
& B_{1}^{\prime}(t)=\mathrm{Z}_{1}(\mathrm{t})+\mathrm{q}_{12}(\mathrm{t}-1) \odot B_{2}^{\prime}(t-1)+\mathrm{q}_{13}(\mathrm{t}-1) \odot B_{3}^{\prime}(t-1)+\mathrm{q}_{14}(\mathrm{t}-1) \odot B_{4}^{\prime}(t-1)+\mathrm{q}_{15}(\mathrm{t}-1) \odot B_{5}^{\prime}(t-1)
\end{aligned}
$$




$$
+\mathrm{q}_{16}(\mathrm{t}-1) \odot B_{6}^{\prime}(t-1) \text {. }
$$

$$
\begin{aligned}
B_{2}^{\prime}(t) & =\mathrm{q}_{20}(\mathrm{t}-1) \odot B_{0}^{\prime}(t-1)+\mathrm{q}_{24}(\mathrm{t}-1) \odot B_{4}^{\prime}(t-1)+\mathrm{q}_{27}(\mathrm{t}-1) \odot B_{7}^{\prime}(t-1) . \\
B_{3}^{\prime}(t) & =\mathrm{q}_{30}(\mathrm{t}-1) \odot B_{0}^{\prime}(t-1)+\mathrm{q}_{35}(\mathrm{t}-1) \odot B_{5}^{\prime}(t-1)+\mathrm{q}_{37}(\mathrm{t}-1) \odot B_{7}^{\prime}(t-1) . \\
B_{4}^{\prime}(t)= & \mathrm{q}_{42}(\mathrm{t}-1) \odot B_{2}^{\prime}(t-1) . \\
B_{5}^{\prime}(t)= & \mathrm{q}_{53}(\mathrm{t}-1) \odot B_{3}^{\prime}(t-1) . \\
B_{6}^{\prime}(t)= & \mathrm{z}_{6}(\mathrm{t})+\mathrm{q}_{61}(\mathrm{t}-1) \odot B_{1}^{\prime}(t-1)+\mathrm{q}_{62}(\mathrm{t}-1) \odot B_{2}^{\prime}(t-1)+\mathrm{q}_{63}(\mathrm{t}-1) \odot B_{3}^{\prime}(t-1)+\mathrm{q}_{64}(\mathrm{t}-1) \odot B_{4}^{\prime}(t-1) \\
& +\mathrm{q}_{65}(\mathrm{t}-1) \odot B_{5}^{\prime}(t-1) . \\
B_{7}^{\prime}(t)= & \mathrm{q}_{70}(\mathrm{t}-1) \odot B_{0}^{\prime}(t-1)+\mathrm{q}_{71}(\mathrm{t}-1) \odot B_{1}^{\prime}(t-1)+\mathrm{q}_{76}(\mathrm{t}-1) \odot B_{6}^{\prime}(t-1)
\end{aligned}
$$

By taking geometric transformation and solving the equation

$$
B_{0}^{\prime}(h)=\frac{N_{4}(h)}{D_{2}(h)}
$$

The probability that the repair facility is busy in repair of failed unit is given by

$$
B_{0}^{\prime}=\lim _{t \rightarrow \infty} B_{0}^{\prime}(t)
$$

Hence, by applying ' $L$ ' Hospital Rule, we get

where

$$
B_{0}^{\prime}=-\frac{\mathrm{N}_{3}(1)}{\mathrm{D}_{2}^{\prime}(1)}
$$

$\mathrm{N}_{4}(1)=\mu_{1}\left[1-\mathrm{P}_{24}-\mathrm{P}_{27} \mathrm{P}_{76}\left(1-\mathrm{P}_{61}\right)\right]+\mu_{6}\left[\mathrm{P}_{16}\left(1-\mathrm{P}_{24}\right)+\mathrm{P}_{27} \mathrm{P}_{76}\left(1-\mathrm{P}_{16}\right)\right]$

and $\mathrm{D}_{2}^{\prime}(1)$ is the same as in availability analysis.

\section{PROFIT FUNCTION ANALYSIS}

The expected total profit in steady-state is

$$
\mathrm{P}=\mathrm{C}_{0} \mathrm{~A}_{0}-\mathrm{C}_{1} \mathrm{~B}_{0}-\mathrm{C}_{2} B_{0}^{\prime}
$$

where

$\mathrm{C}_{0}$ : be the per unit up time revenue by the system

$\mathrm{C}_{1} \& \mathrm{C}_{2}$ : be the per unit down time expenditure on the system

\section{GRAPHICAL REPRESENTATION}

The behaviour of the MTSF and the profit function w.r.t failure rate and repair rate have been studied through graphs by fixing the values of certain parameters $\mathrm{a}, \mathrm{b}, \mathrm{C}_{0}, \mathrm{C}_{1}$ and $\mathrm{C}_{2}$ as $\mathrm{a}=0.4, \mathrm{~b}=0.6, \mathrm{C}_{0}=2000, \mathrm{C}_{1}=400$ and $\mathrm{C}_{2}=600$.

On the basis of the numerical values taken as:

$\mathrm{P}=1361.562, \mathrm{r}=0.3$ and $\mathrm{s}=0.7$

The values of various measures of system effectiveness are obtained as:

Mean time to system failure $($ MTSF $)=26.662554$.

Availability $\left(\mathbf{A}_{\mathbf{0}}\right)=0.858787$.

Busy period of Inspector $\left(\mathbf{B}_{0}\right)=0.286262$.

Busy period of repairman $\left(B_{0}^{\prime}\right)=0.402512$.

Figure: 2 show the behavior of MTSF w.r.t failure rate $\left(\mathrm{p}_{1}\right)$.It appears from graph that MTSF decreases with increase in failure rate.

Figure: 3 show the behavior of MTSF w.r.t repair rate (r). It appears from graph that MTSF increases with increase in repair rate.

Figure: 4 show the behavior of Profit function w.r.t failure rate $\left(\mathrm{p}_{1}\right)$. It appears from graph that Profit function decreases with increase in failure rate.

Figure: 5 show the behavior of Profit function w.r.t repair rate (r). It appears from graph that Profit funtion increases with increase in repair rate for different 


\section{CONCLUSION}

This paper provided the results for the various reliability measures like MTSF, availability and busy period of repairman and inspector which helps to get the conclusion that the maintenance of units increases both the availability and profit of the system. It also provides information for other researchers and companies following such systems to prefer the equipments which satisfied the conditions as discussed in this paper.

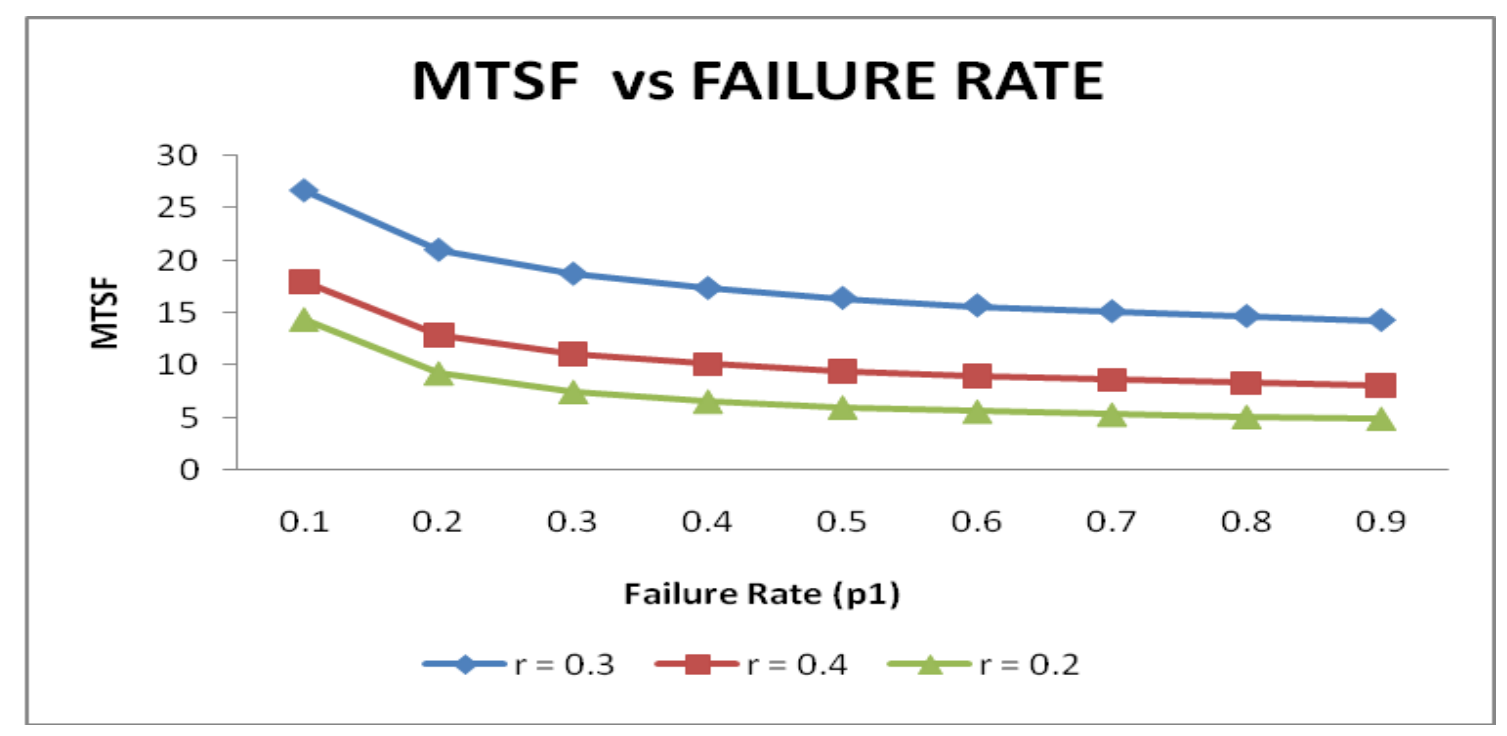

Figure 2: MTSF vs FAILURE RATE

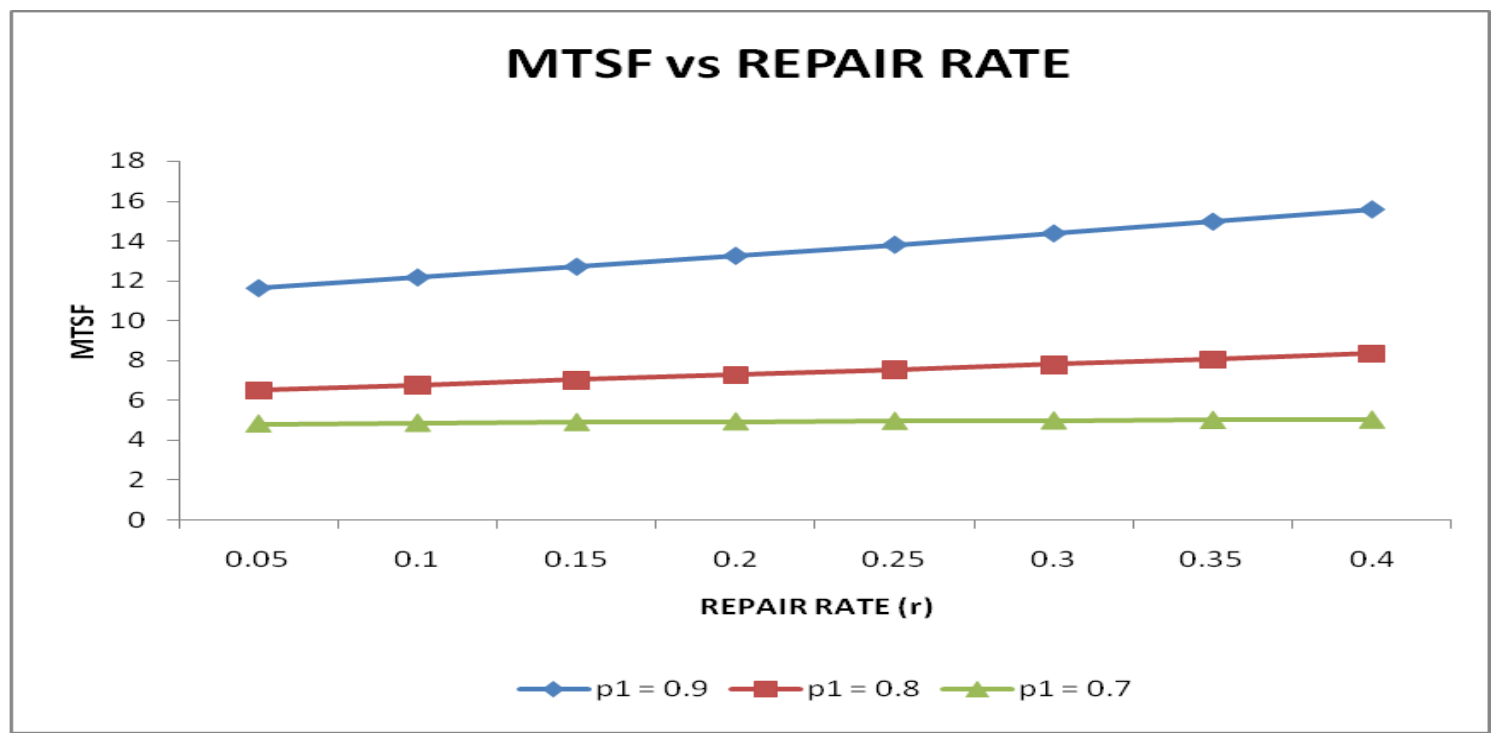

Figure 3: MTSF vs REPAIR RATE 


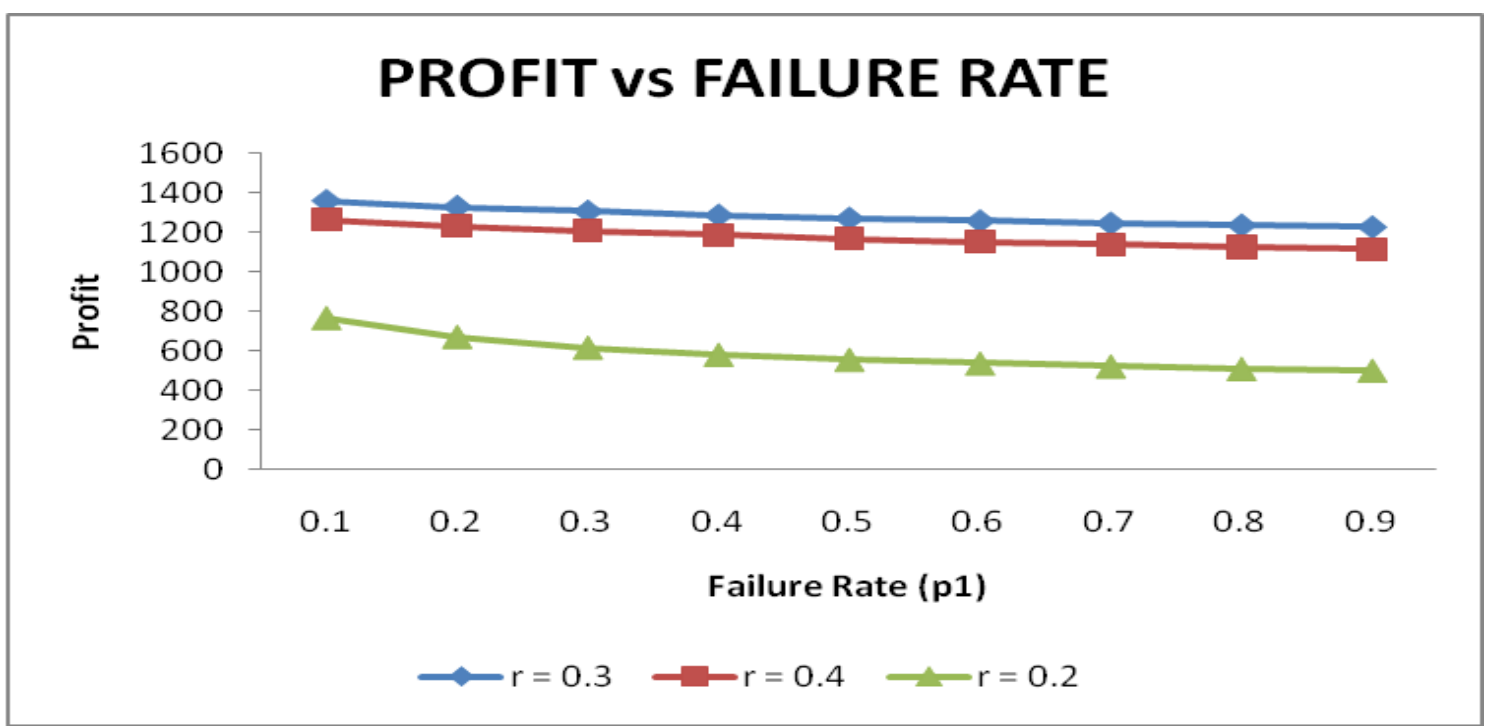

Figure 4: PROFIT vs FAILURE RATE

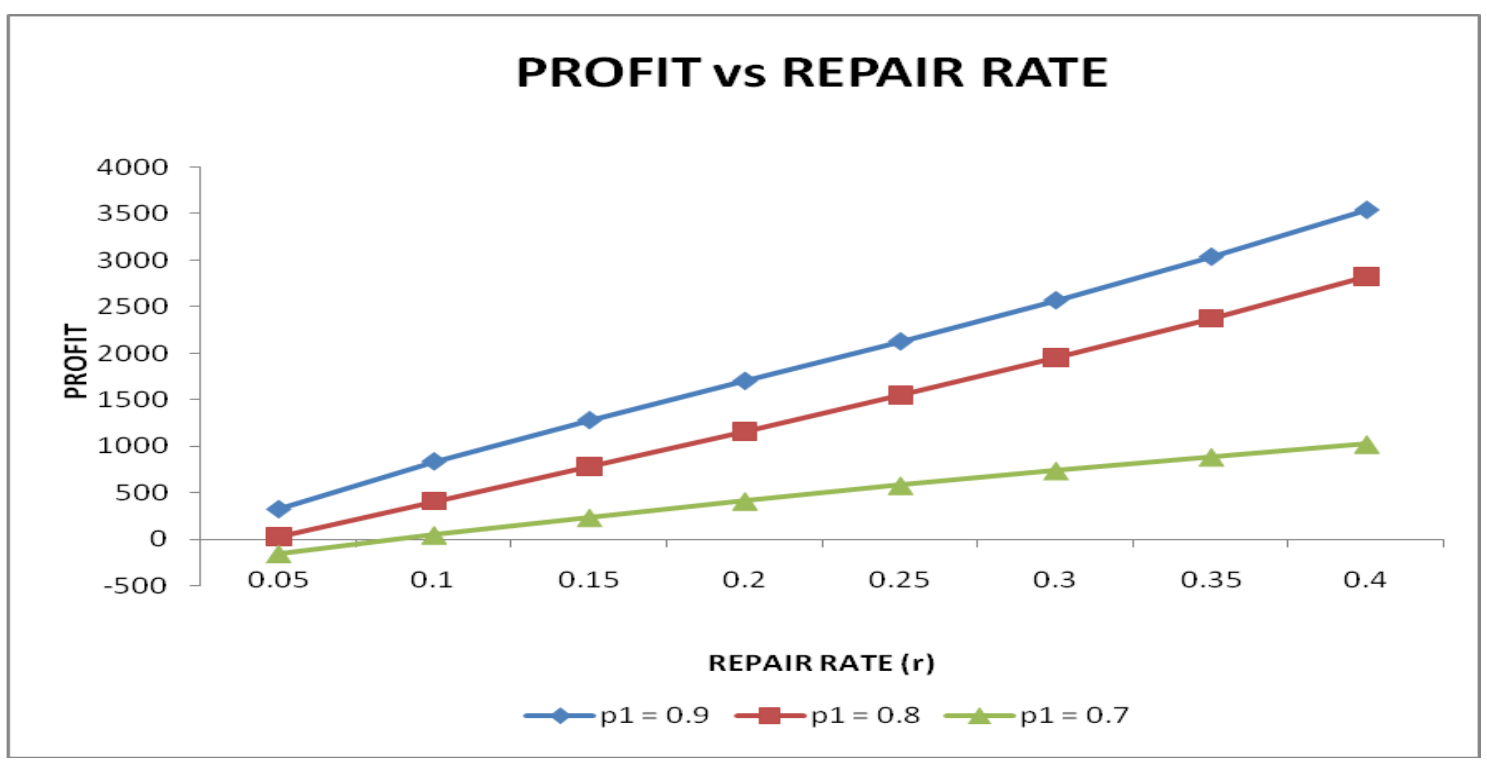

Figure 5: PROFIT vs REPAIR RATE

\section{REFERENCES}

[1] Agarwal S.C, Sahani M, Bansal S (2010) "Reliability characteristic of cold-standby redundant syatem", May 2010 3(2), IJRRAS.

[2] Haggag M.Y (2009) "Cost Analysis of a System Involving Common Cause Failures and Preventive Maintenance", J.Math \& Stat., 5(4):305310, 2009, ISSN 1549-3644.

[3] Haggag. M.Y. (2009), "Cost analysis of two-dissimilar unit cold standby system with three states and preventive maintenance using linear first order differential equations" J.Math \& Stat., 5(4):395-400, 2009, ISSN 1549-3644.

[4] Bhardwaj N (2009) "Analysis of two unit redundant system with imperfect switching and connection time", International transactions in mathematical sciences and Computer,July-Dec. 2009,Vol. 2, No. 2, pp. 195-202.

[5] Bhardwaj N, Kumar A, Kumar S (2008) "Stochastic analysis of a single unit redundant system with two kinds of failure and repairs", Reflections des. ERA-JMS, Vol. 3 Issue 2 (2008), 115-134

[6] Gupta R, Varshney G (2007) "A two identical unit parallel syatem with Geometric failure and repair time distributions", J. of comb. Info. \& System Sciences, Vol. 32, No.1-4, pp 127-136 (2007)

[7] Said K.M.EL, Salah M, Sherbeny EL (2005) "Profit analysis fof a two unit cold standby system with preventive maintenance and random change in Units", 1(1):71-77, 2005, ISSN 1549-3644 (2005). 